\title{
THE EFFECT OF CERAMIC MATERIAL AND PREPARATION DESIGN ON THE FRACTURE RESISTANCE OF ONLAY RESTORATIONS
}

\author{
Hoda Mohamed Abdel Sadek*, Malak Khaled Al-Qatta** and Marwa Mohamed Wahsh ${ }^{* * *}$
}

\begin{abstract}
Objectives: Evaluating the fracture resistance and failure mode of human maxillary premolars restored with two ceramic onlay materials prepared with two preparation designs.

Materials and methods: A total of 40 extracted, human maxillary premolars were selected and divided into two groups according to preparation design; Group A: MOD cavity with anatomical reduction of functional cusp and Group F: MOD cavity with flat reduction of the functional cusp. Each group was divided into two subgroups according to the material of construction; Subgroup S: restored using zirconia-reinforced glass-ceramic and Subgroup E: restored using hybrid ceramic. The finished onlay restorations were luted to prepared teeth using self-adhesive resin cement. Specimens were exposed to thermocycling for 5000 cycles. Fracture resistance was assessed using a Universal testing machine. Failure mode was evaluated using a stereomicroscope. Two-way ANOVA was used to assess the effect of different tested variables and their interaction. Intergroup comparison was done using one-way ANOVA followed by Tukey's post hoc test. The significance level was set at $\mathrm{p} \leq 0.05$ within all tests.
\end{abstract}

Results: Two-Way ANOVA showed that ceramic material had no significant effect on the fracture resistance of restored teeth. While the preparation design had a statistically significant effect. The interactions between the independent variables, ceramic materials, and preparation designs had a significant effect on the fracture resistance of teeth.

Conclusion: Both preparation designs and materials showed clinically acceptable fracture resistance of onlay restorations. All subgroups showed high fracture resistance and favorable failure modes except VITA SUPRINITY with anatomical reduction of the functional cusp.

KEYWORDS: Fracture resistance, onlay restoration, zirconia-reinforced glass-ceramic, hybrid ceramic

\footnotetext{
* Lecturer, Department of Fixed Prosthodontics, Faculty of Dentistry, Ain Shams University

** Master's degree student, Department of Fixed Prosthodontics, Faculty of Dentistry, Ain Shams University

*** Professor, Department of Fixed Prosthodontics, Faculty of Dentistry, Ain Shams University
} 


\section{INTRODUCTION}

Restorative procedures such as decay removal or cavity preparation are accompanied by a reduction in tooth stability, decreased fracture resistance, and increased deflection of weakened cusps. ${ }^{1}$ Different treatment options are available, depending on the degree of destruction either direct restoration with composite or partial indirect restoration. The main determinants in fracture are the restorative material and the geometry of cavity preparation. Cuspal coverage has been known to greatly affect the fracture resistance of teeth restored with onlay restorations. Numerous designs have been suggested for preparing all-ceramic onlays, as influenced by the mechanical and structural qualities of ceramic materials. ${ }^{2}$ Anatomical preparation design was suggested to reduce the loss of healthy tooth tissue and decrease dentin exposure areas and to define the margin design which contributes to the quality of the adhesion, enhancing the cutting of the enamel prisms and increasing enamel surface area. In addition, the anatomic preparation design improves the insertion of the restoration during cementation and improve the esthetic outcome between the tooth and the restoration. ${ }^{3}$ Flat preparation design can help to transform tensile into compressive stresses. The design also helps to avoid stress peaks and material collections where smooth transitions at flat edges can reduce stress build-up., ${ }^{4,5}$ Due to the high physical properties of indirect restorations, in case of large destruction in tooth structure, they are mostly preferred over direct restorations. ${ }^{6}$ Besides, when the restoration is too difficult to make directly as in cases of cusp fracture and large defective size, or when optimal form and esthetics are required, an indirect restoration can be more successful. In general, indirect techniques have many advantages as expected to have better longevity than direct restorations. $^{7}$

The use of ceramics with adhesive techniques permits the preservation of tooth structure and more esthetic restorations in posterior teeth. ${ }^{8}$ However, the fracture of bonded ceramics becomes a concern when considering the same treatments for posterior teeth. This is particularly the case with restorations covering the cusps like onlays. Computer-aided design /Computer-aided manufacturer (CAD/ CAM) technology has recently viewed a noticed development in the range of available materials for dental use. ${ }^{7}$ Thus far, lithium disilicate CAD blocks appear to be an attractive monolithic material that can be used in inlays, onlays and, overlays.

Recently, $10 \%$ by weight zirconia has been added to lithium silicate ceramic referred to as zirconia reinforced lithium silicate or ZLS). ${ }^{9}$ The incorporation of zirconia filler reinforces the glass matrix without being packed by the dissolved zirconia particles, which gives it higher fracture toughness of the material. ${ }^{10}$

Another approach for an optimized CAD/CAM material is a polymer-infiltrated ceramic network (PICN), which consists of 86 wt. \% ceramic and 14wt. \% polymers. The material exhibits similar elastic properties to teeth as a result, the occlusal load compensation capacity of hybrid ceramic is higher than traditional ceramics. ${ }^{9}$ The null hypothesis was that the preparation design and ceramic material would have no effect on the fracture resistance of onlay restorations.

\section{MATERIAL AND METHODS}

\section{Teeth selection}

Forty sound freshly extracted maxillary premolars free of carious lesions and cracks were selected for this study. All external debris were removed with an ultrasonic scaler and teeth were stored in saline solution. The dimensions of the crown of all teeth were measured using a digital caliper (digital caliper, Hogetex). The mean dimensions of teeth were: $7.06 \mathrm{~mm}$ (mesiodistally) and $9.24 \mathrm{~mm}$ (buccopalatally). The roots of all teeth were dipped into 
molten wax (dipping wax) up to $2 \mathrm{~mm}$ below the cementoenamel junction to simulate the periodontal ligament, resulting in a uniform wax layer of $0.2 \mathrm{~mm}$ thickness, then the roots were embedded in epoxy resin using a dental surveyor. A silicone-based light body impression material was injected into the epoxy resin blocks after the wax was molten and the teeth were reinserted into the epoxy blocks.

\section{Samples grouping}

Teeth were divided into two groups $(n=20)$ according to preparation designs, group A: MOD cavity with anatomical reduction of functional cusp, group F: MOD cavity with flat reduction of the functional cusp, then each group was divided into two subgroups according to the material of construction of onlay restoration $(n=10)$, subgroup $\mathrm{S}$ : restored using zirconia-reinforced glass-ceramic (VITA SUPRINITY) and subgroup E: restored using hybrid ceramic (VITA ENAMIC).

\section{Teeth Preparation}

Teeth preparations were made by the same operator with the recommended sequence of specific diamond burs (Fg Ser- inlay set III Extended, I. Sevuk, Istanbul). The prepared cavity was $2 \mathrm{~mm}$ indepth at the central groove, the isthmus width was $2 \mathrm{~mm}, 6$ occlusal tapers of axial, and 90 cavo-surface margins. The gingival seat of the proximal boxes was prepared $1.5 \mathrm{~mm}$ below the pulpal floor. Cusp reduction for palatal cusps was $2 \mathrm{~mm}$ in flat occlusal reduction at the cusp tip in half of the samples and 2 $\mathrm{mm}$ even reduction at the cusp tip and cusp slope in anatomical design in another half of samples (figure 1 and figure 2).

\section{Restorations fabrication and cementation}

Each prepared tooth was individually scanned indirectly by a desktop extraoral scanner (E2 Lab scanner, 3 shapes, Copenhagen, Denmark). The digital data from the scanning process was used to create virtual dies using CAD system software.
Exocad (GmbH, Darmstadt, Germany) in-lab system software design was used to design the restoration. Milling orders were given for each restoration in CAD/CAM unit (CORiTECH 250i, Imes-icore $\mathrm{GmbH}$, Eiterfeld, Germany) to produce 20 zirconiareinforced glass-ceramic (VITA SUPRINITY) and 20 hybrid ceramic (VITA ENAMIC) onlay restorations (10 for each preparation design). The polymer infiltrated ceramic restorations (VITA ENAMIC) were polished according to the manufacturer's instructions using a specific polishing set (VITA ENAMIC Polishing Set Vita Zahnfabrik, Bad Säckingen, Germany). Whereas for VITA SUPRINITY restorations, the milled restorations were smoothened using fine grit diamond stone under water coolant, and before crystallization then the restorations were cleaned using the ultrasonic cleaner for 5 minutes. VITA firing paste was applied to the external surface of each restoration and positioned on the firing try and crystalized using (Programat P510 Ivoclar Vivadent). All prepared teeth were selectively etched with $37 \%$ phosphoric acid for 20 seconds on the enamel margin. The inner surface of each onlay restoration was etched according to the manufacturer's instruction using 9.5\% hydrofluoric acid (Bisco Inc, Schaumburg, I1, USA) for 30 seconds in VITA ENAMIC and 20 seconds in VITA SUPRINITY then rinsed and silane was applied. The Self-adhesive Duo-Link resin cement (Aureocem NE) was used for cementation of the onlay ceramic samples to the prepared teeth. A loading device was used to apply a standardized load of $1 \mathrm{Kg}$ directly towards the central groove, then samples were exposed to a brief light curing for only 2 seconds using 3M ESPE curing light with a wavelength range between $450-470 \mathrm{~nm}$ and the excess cement was removed with an explorer, and then light-curing was done for 20 seconds for each side. ${ }^{4}$ The teeth were subjected to thermocycling using Robota thermocycler (Alexandria, Egypt) for 5000 cycles between $5 \pm 2^{\circ} \mathrm{C}$ and $55 \pm 2^{\circ} \mathrm{C}$ with a dwell time of 30 seconds in each bath and $20 \mathrm{sec}$ interval between baths at Ambient air. 


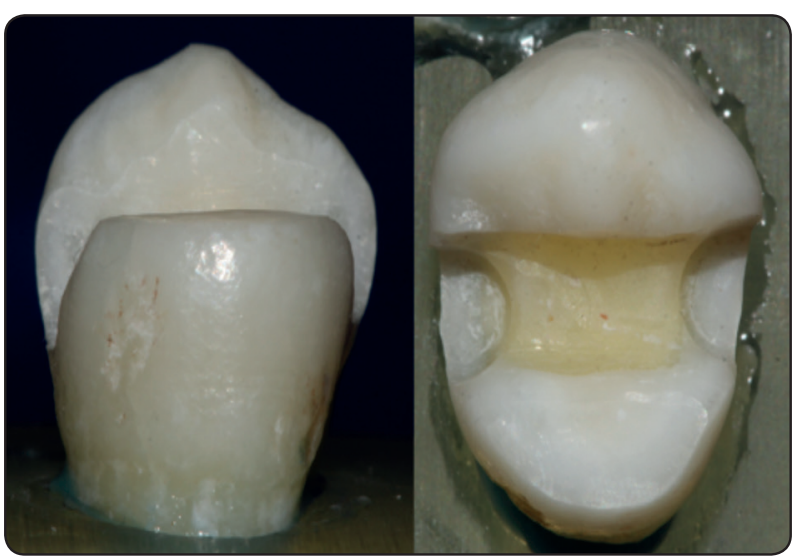

Fig. (1) MOD cavity with flat reduction of functional cusp.

\section{Fracture Resistance test}

All samples were individually mounted on a computer-controlled material testing machine (Model 3345; Instron Industrial Products, Norwood, MA, USA) with a loadcell of $5 \mathrm{kN}$, and data were recorded using computer software (Instron ${ }^{\circledR}$ Bluehill Lite Software). The compressive mode of load was applied occlusally using a metallic rod with a round tip (3.8 $\mathrm{mm}$ diameter) attached to the upper movable compartment of the testing machine traveling at a crosshead speed of $1 \mathrm{~mm} / \mathrm{min}$ with a tin foil sheet in between to ensure homogenous stress distribution. The failure load recorded in Newton was manifested by an audible crack and confirmed by a sharp drop at load-deflection curve.

Failure mode was evaluated using a stereomicroscope. The failure mode was assessed based on the previous publication by Alassar et al ${ }^{11}$ as follows: Type (I): restoration fracture, Type (II): Restorable tooth fracture, including cracks and/or cusp fractures, horizontal fractures, oblique fractures not reaching the CEJ, Type (III): Unrestorable tooth fractures, including vertical fractures or oblique fractures violating the CEJ, and Type (IV): A combined fracture in both tooth and restoration. Twoway ANOVA was used to study the effect of different tested variables and their interaction. Intergroup

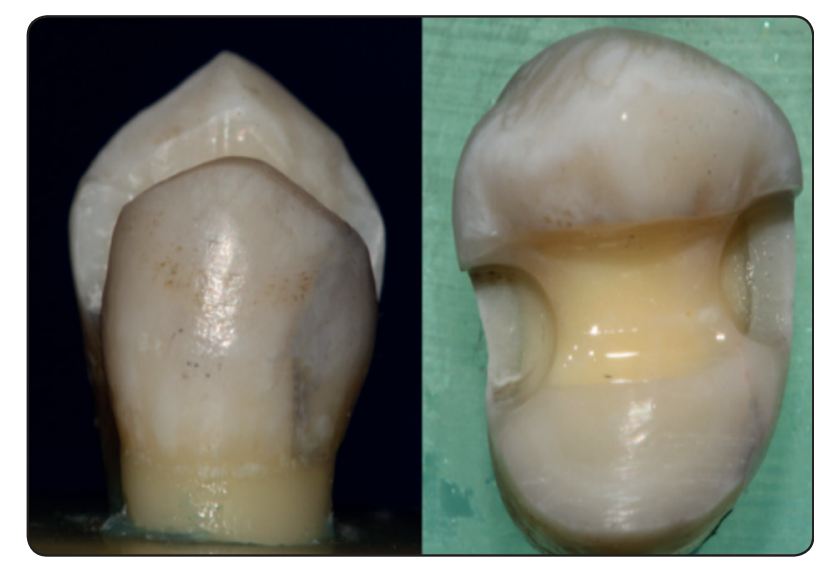

Fig. (2) MOD cavity with anatomical reduction of functional cusp.

comparison was done using one-way ANOVA followed by Tukey's post hoc test. The significance level was set at $\mathrm{p} \leq 0.05$ within all tests.

\section{RESULTS}

Mean and standard deviation values of fracture resistance for different groups were presented in Table 2. Two-Way ANOVA (Table 1) showed that ceramic material had no significant effect on the fracture resistance of restored teeth. On the other hand, the preparation design had statistically significant effect. The interactions between the independent variables, ceramic material and preparation design had significant effect on the fracture resistance of restored teeth. There was a significant difference between different groups $(\mathrm{p}<0.001)$. The highest value was found in VITA SUPRINITY samples made with flat occlusal preparation $(882.55 \pm 94.19)$ followed by VITA ENAMIC samples with the flat occlusal preparation $(819.56 \pm 92.50)$, then samples of VITA ENAMIC made with anatomical preparation $(791.53 \pm 80.52)$ while the lowest value was found in VITA SUPRINITY samples made with anatomical preparation $(666.15 \pm 79.52)$. Post hoc pairwise comparisons showed value of VITA SUPRINITY samples with anatomical preparation to have a significantly lower value than all other samples $(\mathrm{p}<0.001)$. The failure mode analysis is presented in Table 3 and Figure 3. 
TABLE (1): Effect of different variables and their interactions on fracture resistance $(\mathrm{N})$

\begin{tabular}{|l|c|c|c|c|c|}
\hline \multicolumn{1}{|c|}{ Source } & Sum of squares & df & Mean square & f-value & p-value \\
\hline Material & 9729.72 & 1 & 9729.72 & 1.29 & $\mathbf{0 . 2 4 6 n s}$ \\
\hline Preparation design & 149368.73 & 1 & 149368.73 & 19.76 & $<\mathbf{0 . 0 0 1 *}$ \\
\hline Material * Preparation design & 88709.08 & 1 & 88709.08 & 11.74 & $\mathbf{0 . 0 0 2} *$ \\
\hline
\end{tabular}

$d f=$ degree of freedom*; significant $(p \leq 0.05) n s ;$ non-significant $(p>0.05)$

TABLE (2): Mean \pm standard deviation (SD) of fracture resistance $(\mathrm{N})$ for different preparation designs:

\begin{tabular}{|c|c|c|c|c|}
\hline Groups & Type I & Type II & Type III & Type IV \\
\hline AE & 4 & 1 & 2 & 4 \\
\hline AS & 1 & 0 & 3 & 0 \\
\hline FE & 3 & 4 & 2 & 1 \\
\hline
\end{tabular}

TABLE (3): Number of samples for different fracture patterns of tested groups:

Anatomical preparation

Vita Enamic (AE)

$791.53 \pm 80.52^{\mathrm{A}}$

Vita Suprinity (AS)

$666.15 \pm 79.52^{\mathrm{B}}$
Flat preparation

$\begin{array}{ccc}\text { Vita Enamic (FE) } & \text { Vita Suprinity (FS) } & \text { p-value } \\ 819.56 \pm 92.50^{\mathrm{A}} & 882.55 \pm 94.19^{\mathrm{A}} & <\mathbf{0 . 0 0 1 *}\end{array}$

Means with different superscript letters within the same horizontal raw show statistically significant difference *; significant $(p \leq 0.05)$ ns; non-significant $(p>0.05)$.
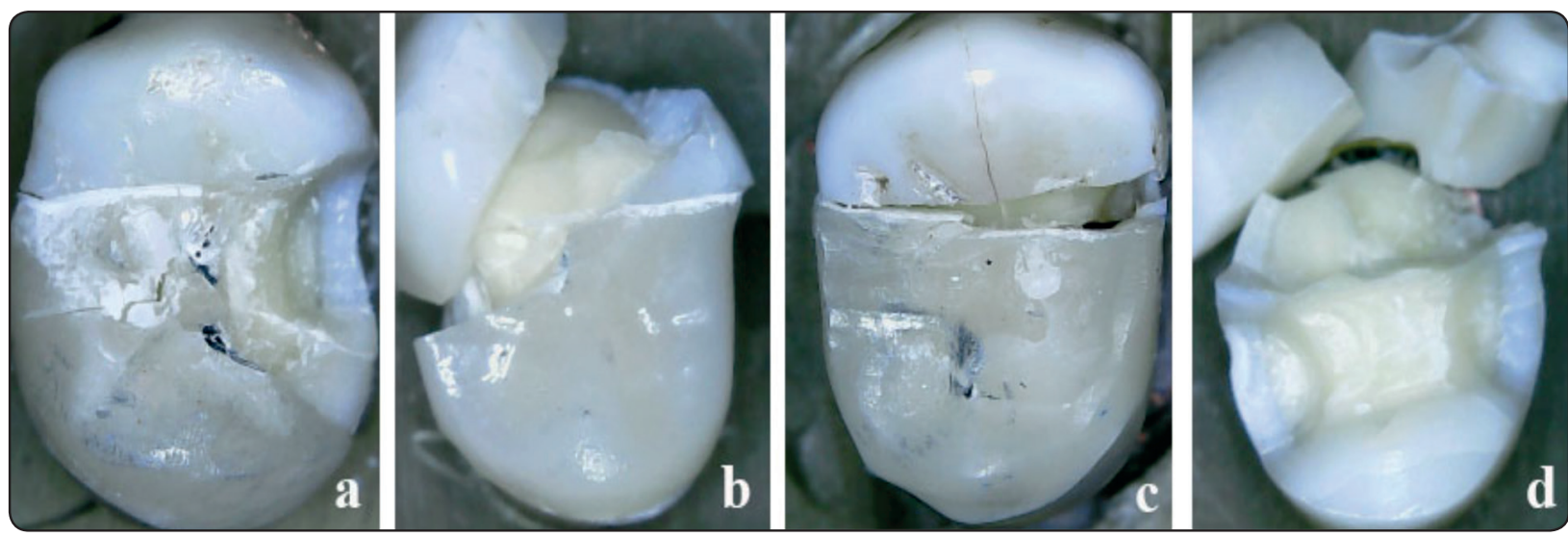

Fig. (3) Stereomicroscopic photos showing fracture patterns of tasted group a: type I, b: type II, c: type III, d: type IV. 


\section{DISCUSSION}

The use of tooth-colored ceramic restorative materials has increased significantly in the last decade. With the development and improvement of reliable adhesive bonding techniques, minimally invasive dentistry has become a field of great interest in modern restorative dentistry. ${ }^{6}$ Preserving tooth structure is critical for the longevity of teeth and restorations. ${ }^{1,12}$ One of the minimal invasive concept applications is the partial coverage restorations. Whenever indicated, these restoration designs offer several advantages over the full coverage restorations including ease in maintaining excellent periodontal health. Besides cementation can be done with less hydrolytic behavior, pulp's health and tooth's anatomical shape are preserved. However, previous research addressed the ceramic bulk fracture as the most common failure type for ceramic partial coverage restorations. ${ }^{13}$

This study was performed on maxillary natural human extracted premolars. Since the anatomy of maxillary premolars is susceptible to cusp deflection and fracture under excessive occlusal forces. It is necessary to protect cusps when the width of the cavity isthmus is greater than two-third of the inter-cuspal distance or half of the buccolingual distance. ${ }^{11}$

The preparation design used in the current study was based on a MOD onlay cavity with cusp reduction for palatal cusps was $2 \mathrm{~mm}$ with flat design and anatomical design. ${ }^{14}$ The anatomical type preparation design was a conservative preparation that over-wrapped or capped the cusps. ${ }^{3}$ On the other hand, the flat preparation type preserved the outer cuspal slopes but preserved less of the cusp tips. Apart from that, the flat preparation was easier to design, prepare and mill..$^{5,15}$

All cemented specimens were preserved in distilled water at $37^{\circ} \mathrm{C}$ for 24 hours till the beginning of the test. For simulation of intraoral conditions, they were subjected to thermocycling (Theromocycler, Robota, Alexandria, Egypt) for a total number of 5000 cycles between $5^{\circ} \mathrm{C}$ and $55^{\circ} \mathrm{C}$. Thermal cycling tests are intended to produce alternate stresses at the interface of different materials based upon temperature changes. ${ }^{16}$ The difference in the thermal expansion coefficients of the materials causes adhesive failures under temperature variations.${ }^{17}$ The conventional methods used for evaluating the fracture load of ceramics might fail to reflect their real clinical behavior. Thus, thermal cycling tests are needed to investigate the mechanical properties of ceramic materials. ${ }^{16}$

The fracture resistance values recorded in this study ranged from 666 to $882 \mathrm{~N}$. In the clinical literature, it was reported the normal force at the premolar region varies from 222 to $445 \mathrm{~N}$ but from 520 to $800 \mathrm{~N}$ during clenching. ${ }^{14}$ Therefore, these results are within the clinically accepted limit. According to the results of the study preparation design significantly affected values of fracture strength but ceramic material did not, so the null hypothesis would be partially rejected. The results of this study showed that the preparation design had a statistically significant effect as the fracture resistance of the flat preparation group was higher than that of the anatomical group with the VITA SUPRINITY subgroup. The flat preparation design provides a stable surface that resists the compressive stresses because it is prepared parallel to the occlusal plane. From a biomechanical standpoint, the restoration allows adaptation to strain at the bonded joint which led to increase fracture resistance of the flat occlusal preparation. ${ }^{3,7}$ Besides that, the flat preparation design has less stress concentration than anatomical preparation because of the increase in the thickness of the restoration. ${ }^{4,18}$ Furthermore, the anatomical design is more wedge-shaped than the flat design which produced higher frequency of fracture. ${ }^{5,19}$

The results of this study were in agreement with those of Oyar and Durkan $2018^{20}$ who found that specimens with flat preparation designs had higher mean fracture resistance values than those with anatomic preparation designs; however, it was showed that the results were not statistically significant. 
On the other hand, Alassar et $\mathrm{al}^{7}$ found that anatomical occlusal reduction yielded higher fracture resistance compared to flat occlusal reduction. These differences could be attributed to different restorative materials used to construct overlays (composite) which had a greater ability to absorb impacts than ceramics. On the contrary, these findings are inconsistent with those of Al Khalifah, ${ }^{5}$ in which the different overlay preparations had no effect on fracture resistance. This difference could be explained by the different restorative materials used to construct overlays on molars, and the use of premolars in the current study. Also, Fonseca et, al. ${ }^{21}$ found that the differences in cavity preparation design had no effect on the fracture strength of teeth restored with a laboratory-processed composite resin, but that intact teeth were consistently more resistant to fracture than prepared teeth with all restoration types. The difference in cavity preparation designs as different cusp coverage patterns were evaluated could be the reason for such disagreement in the results of fracture resistance.

In this study, two-way ANOVA showed no significant difference between samples made with the two selected materials $(\mathrm{p}=0.246)$. VITA ENAMIC (polymer infiltrated ceramic network) (PICN) is a hybrid dental ceramic with a dual-network structure. Where predominant ceramic network reinforced by a specific structure called polymer network so it mixes between the best properties of ceramic and a composite together ${ }^{22}$. Moreover to the high degree of elasticity, hybrid ceramics confirms high strength after adhesive bonding, therefore allowing the decrease of the restoration thickness ${ }^{21}$. While Zirconia-reinforced lithium silicate (ZLS) ceramics is enriched with zirconia particles which are incorporated in order to reinforce the ceramic structure by crack interruption which enhanced the mechanical properties. A previous study by Sieper et al ${ }^{9}$ proved that polymer-infiltrated-ceramic-network material and zirconia reinforced lithium silicate ceramic can be a substitute to lithium disilicate ceramic regarding its fracture strength.
These results were in agreement with Hany and Taymour $2017^{13}$ who evaluated the fracture resistance and investigated the failure mode of two restoration designs (crowns and inlays) made from lithium disilicate glass-ceramic and hybrid ceramic and concluded that there was no statistically significant difference between (e.max CAD) and (VITA ENAMIC) inlays. In addition, Zhang et al in $2015^{24}$ conducted a study on glass and hybrid ceramic crowns and found no significant difference between the materials in terms of fracture resistance. However, another study carried out by Abo El-Farag and Elnawawy $2019,{ }^{22}$ who assessed the fracture resistance and modes of failure of composite, ceramic and hybrid partial coverage restorations with different preparations. Statistically significant differences were observed between the three restorative materials in both preparation designs. The reason for the different results may be due to a difference in the preparation design of onlay restoration.

In this study, there was a statistically significant difference between anatomical and flat occlusal preparation design when VITA SUPRINITY was used as a material for fabrication of onlay restoration although there was not statistically significant difference between the preparation designs with VITA ENAMIC. This may be due to the mechanical properties of the materials selected to restore a tooth, that can influence the behavior of stress distribution at the tooth/restoration interface..$^{25}$ As a result of stress concentration associated with anatomical preparation so it affects the brittle ceramic material rather than more resilient hybrid ceramic.

On examination of fractured samples, it was clearly observed that the most common failure mode of the teeth restored by VITA SUPRINITY was unfavorable fracture (i.e., un-restorable) with the anatomical preparation, whereas restorable failure was the predominant in VITA ENAMIC subgroups. This may be related to the high Elastic Modulus of VITA SUPRINITY (zirconia reinforced lithium silicate) $;^{10}$ in which stresses are transmitted to the underlying tooth structure that leads to unfavorable failure. 
In contrast, the composition of VITA ENAMIC (polymer infiltrated ceramic) allows the material to have modulus of elasticity similar to that of dentin ${ }^{9}$ that absorbs forces and equally distributes stresses, leading to favorable failure mode.

This study has several limitations, namely, only one type of cement was examined. Moreover, clinical conditions such as cycling fatigue and accumulated damage from stress and water were not accurately represented. Thus, further studies are required to address these issues.

\section{CONCLUSION}

Within the limitation of this in vitro study, the following could be concluded:

1. Preparation design affected the fracture resistance of onlay restorations where the flat preparation of functional cusp improved the fracture resistance.

2. Both preparation designs and materials showed clinically acceptable fracture resistance of onlay restorations.

3. VITA ENAMIC showed high fracture resistance and favorable modes of failure of onlay restorations with both preparation designs.

4. On using VITA SUPRINITY for onlay restorations, flat occlusal preparation provided higher fracture resistance with more favorable fracture mode.

\section{REFERENCES}

1. Politano G, Fabianelli A, Papacchini F, Cerutti A. The use of bonded partial ceramic restorations to recover heavily compromised teeth. Int J Esthet Dent. 2016;11(3):314-336.

2. Momoi Y, Hayashi M, Fujitani M, Fukushima M, Imazato S, Kubo S, Nikaido T, Shimizu A, Unemori M, Yamaki C. Clinical guidelines for treating caries in adults following a minimal intervention policy - evidence and consensus based report. J Dent. 2012;40(2):95-105.

3. Veneziani M. Posterior indirect adhesive restorations : updated indications and the Morphology Driven Preparation Technique. Int J Esthet Dent,. 2017;12(2):204-230.
4. Arnetzl GV, Arnetzl G. Design of preparations for all-ceramic inlay materials. Int J Comput Dent 2006;9:289-298.

5. Al Khalifah SAM. The Influence of Material Type, Preparation Design and Tooth Substrate on Fracture Resistance of Molar Onlays. UCLA Electronic Theses and Dissertations 2016; 12-41.

6. Amesti-garaizabal A, Agustín-Panadero R, Verdejo-Solá B, Fons-Font A, Fernández-Estevan L, Montiel-Company J, Fernanda M, Solá-Ruíz MF. Fracture Resistance of Partial Indirect Restorations Made with CAD / CAM Technology. A Systematic Review and Meta-Analysis. J Clin Med. 2019;8(11):1-11

7. Alassar RM, Samy A, Abdel-Rahman FM. Effect of cavity design and material type on fracture resistance and failure pattern of molars restored by computer - aided design / computer - aided manufacturing inlays / onlays. Dent Res J (Isfahan). 2021;18(14):1-9.

8. Taha, D., Spintzyk, S., Sabet, A., Wahsh, M. and Salah T. Assessment of marginal adaptation and fracture resistance of endocrown restorations utilizing different machinable blocks subjected to thermomechanical aging. J Esthet Restor Dent. 2018;30(4):319-328.

9. Sieper K, Wille S, Kern M. Fracture strength of lithium disilicate crowns compared to polymer infiltrated ceramic network and zirconia reinforced lithium silicate crowns J Mech Behav Biomed Mater. 2017;74:342-348.

10. Elsaka SE, Elnaghy AM. Mechanical properties of zirconia reinforced lithium silicate glass-ceramic. Dent Mater 2016 Jul; 32(7):908-14

11. Alassar R, Samy A, Amin R. Effect of Cavity Design and Cusp Inclination on Fracture Resistance of Indirect Overlay Restorations In Maxillary First Pemolars: An In Vitro Study. Assiut Dent J. 2020;3(1):7-18.

12. Guess P, Schultheis S, Zang Y, Strub J. Influence of preparation design and ceramic thicknesses on fracture resistance and failure modes of premolar partial coverage restorations. J Prosthet Dent. 2013;110(4):264-273.

13. Hany C, Taymour M. Fracture resistance and failure mode of two restoration designs made of monolithic hybrid and glass machinable ceramics ; in vitro study. Egypt Dent J. 2017;63:2771-2783

14. Yildiz C, Vanlioğlu BA, Evren B, Uludamar A, KulakOzkan Y. Fracture resistance of manually and CAD/ CAM manufactured ceramic onlays. J Prosthodont. 2013; 22(7):537-542. 
15. Arora A, Upadhyaya V, Arora SJ, Jain P, Yadav A. Evaluation of fracture resistance of ceramic veneers with different preparation designs and loading conditions: An in vitro study. J Indian Prosthodont. 2017;17(4):325-331.

16. Yang R, Arola D, Han Z, Zhang X. A comparison of the fracture resistance of three machinable ceramics after thermal and mechanical fatigue. J Prosthet Dent. 2000;112(4): 878-885.

17. Vásquez V, Özcan M, Nishioka R, Souza R, Mesquita A, Pavanelli C. Mechanical and Thermal Cycling Effects on the Flexural Strength of Glass Ceramics Fused to Titanium. Dent Mater J. 2008;27(1):7-15.

18. Oyar P, Ulusoy M. Finite element analysis of stress distribution in ceramic crowns fabricated with different tooth preparation designs. J Prosthet Dent. 2014; 112(4):871-877.

19. Falahchai M, Hemmati YB, Asli HN, Rezaei E. Effect of Tooth Preparation Design on Fracture Resistance of Zirconia-Reinforced Lithium Silicate Overlays. J Prosthodont. 2020;29(7):1-6.

20. Oyar P, Durkan R. Effect of Cavity Design on the Fracture
Resistance of Zirconia Onlay Ceramics. Niger J Clin Pract. 2018;21(6):687-691.

21. Fonseca RB, Correr-Sobrinho L, Fernandes- Neto AJ, Quagliatto PS, Soares CJ. The influence of cavity preparation design on fracture strength and mode of fracture of laboratory-processed composite resin restorations. J Prosthet Dent. 2007;98(4):277-248.

22. Abo El-Farag SA, El Nawawy MS. Fracture resistance of different esthetic partial coverage restorations. Egypt Dent J. 2019;65:599-609.

23. Emam ZN, A.Aleem NA. Influence of different materials and preparation designs on marginal adaptation and fracture resistance of CAD/CAM fabricated occlusal veneers. Egypt Dent J. 2020;66:439-452.

24. Zhang Y, Mai Z, Barani A, Bush M, Lawn B. Fractureresistant monolithic dental crowns. Dent Mater. 2016; 32(2):442-449.

25. Clausen J, Tara M, Kern M. Dynamic fatigue and fracture resistance of non-retentive all-ceramic full-coverage molar restorations. Influence of ceramic material and preparation design. Dent Mater. 2010;26(6):533-538. 